\title{
DFT Analysis of Quantum Transport in Si Atom Wire under Finite Bias Voltage*
}

\author{
Hiroyuki Kusaka ${ }^{\dagger}$ and Nobuhiko Kobayashi \\ Institute of Applied Physics, University of Tsukuba, \\ 1-1-1 Tennodai, Tsukuba, Ibaraki 305-8573, Japan
}

(Received 10 November 2008; Accepted 25 December 2008; Published 10 January 2009)

\begin{abstract}
We have theoretically analyzed electron transport in a Si atom wire at a finite bias voltage using a first principles method. Electronic states and transport properties are calculated using the Lippmann-Schwinger equation using the Laue representation in the framework of the density functional theory. We analyzed the transport properties of the $\mathrm{Si}$ wires between the metallic electrodes, and elucidated the effects of metallic contacts on Si atom wire, the character of conduction channels, and the dependence on the bias voltage. The conduction channels are analyzed using eigen channel decompositions and then it is found that the three channels contributing to the transport are open in the bias window under the finite bias voltage. [DOI: 10.1380/ejssnt.2009.17]
\end{abstract}

Keywords: Density functional calculations; Quantum conductance; Surface electrical transport; Silicon; Metal-semiconductor interfaces; Nano-wires; Quantum wires

\section{INTRODUCTION}

Experimental techniques continue the remarkable development recently, and a great variety of atomic-scale structures can be fabricated. Quantum transport properties of atomic-scale contacts and atomic wires have been intensively investigated experimentally using scanning tunneling microscopy (STM) and mechanically controllable breaking junctions (MCBJ) and so on [1]. Silicon is one of the most important materials from the viewpoint of nanoscale electronics, and transport properties of atomics-scale contacts for silicon has been investigated using high resolution transmission electron microscopy [2] and STM [3]. Theoretically, transport properties of Si atom wires have been analyzed at the zero-bias limit using density functional calculations $[4,5]$.

We report a theoretical analysis of electron transport in a $\mathrm{Si}$ atom wire at a finite bias voltage using a firstprinciples method. We consider a Si atom wire sandwiched between semi-infinite metallic jellium electrodes, and the electronic states and the transport properties are calculated using the Lippmann-Schwinger equation [6]. The equation is solved in the Laue representation, where wave functions are expressed using the two-dimensional plane wave expansion and the one-dimensional real space grid. We analyze the transport properties of the Si wires between the metallic electrodes, and elucidate the effects of metallic contacts on $\mathrm{Si}$ atom wire, the character of conduction channels, and the dependence on the bias voltage. The conduction channels are analyzed using eigen channel decompositions [6] and we found that the three channels contributing to the transport are open in the bias window under finite bias voltage.

\section{METHOD OF CALCULATION}

We have investigated the electronic states and the transport properties of an atomic wires at a finite bias

* This paper was presented at International Symposium on Surface Science and Nanotechnology (ISSS-5), Waseda University, Japan, 9-13 November, 2008.

†Corresponding author: bk200730353@s.bk.tsukuba.ac.jp voltage via the density functional method. The details of the method are described in Ref. [6]. An atomic wire sandwiched between semi-infinite metallic jellium electrodes with different Fermi energies is considered. The $z$ axis is set normal to the surface of the jellium electrodes, and a supercell structure is taken in $x$ and $y$ directions. The positive back ground density of jellium $\rho^{+}$ is expressed by $r_{s}=2.0$ bohrs, where $\frac{4}{3} \pi r_{s}^{3}=\frac{1}{\rho^{+}}$. The bond length of Si atoms is 4.4 bohrs, and the atom-jellium distance is 2.3 bohrs. The side length of the super cell in the $x$ and $y$ directions is 12 bohrs. Wave functions are expressed in the Laue representation, and the LippmannSchwinger equation is solved using expansion with twodimensional plane waves and the one-dimensional real space grid [7]. The wave function $\Psi(\boldsymbol{r})$ for the total system is obtained using the Green function $g\left(\boldsymbol{r}, \boldsymbol{r}^{\prime}\right)$ and the wave functions $\Psi^{0}(\boldsymbol{r})$ for the system without atoms.

The Hamiltonian of the system composed of two semiinfinite jellium electrodes without atoms is

$$
\mathcal{H}^{0}=-\frac{\hbar^{2}}{2 m} \nabla^{2}+v_{\text {eff }}^{0}(\boldsymbol{r}),
$$

where the effective potential is a sum of the Hartree, and exchange-correlation potentials:

$$
v_{\mathrm{eff}}^{0}(\boldsymbol{r})=v_{\mathrm{h}}^{0}(z)+v_{\mathrm{xc}}\left[\rho^{0}(z)\right] .
$$

The Hamiltonian of the total system with atoms is

$$
\mathcal{H}=\mathcal{H}^{0}+\Delta V\left(\boldsymbol{r}, \boldsymbol{r}^{\prime}\right),
$$

where

$$
\begin{aligned}
\Delta V\left(\boldsymbol{r}, \boldsymbol{r}^{\prime}\right)= & v_{\mathrm{eff}}\left(\boldsymbol{r}, \boldsymbol{r}^{\prime}\right)-v_{\mathrm{eff}}^{0}(\boldsymbol{r}), \\
v_{\mathrm{eff}}\left(\boldsymbol{r}, \boldsymbol{r}^{\prime}\right)= & v_{\mathrm{nl}}\left(\boldsymbol{r}, \boldsymbol{r}^{\prime}\right) \\
& +\left\{v_{\mathrm{l}}(\boldsymbol{r})+v_{\mathrm{h}}(\boldsymbol{r})+v_{\mathrm{xc}}[\rho(\boldsymbol{r})]\right\} \delta\left(\boldsymbol{r}-\boldsymbol{r}^{\prime}\right) .
\end{aligned}
$$

Here, $v_{\mathrm{nl}}\left(\boldsymbol{r}, \boldsymbol{r}^{\prime}\right)$ and $v_{\mathrm{l}}(\boldsymbol{r})$ are nonlocal and local parts of atomic pseudopotentials. Two different Fermi energies $E_{F}^{L}$ and $E_{F}^{R}$ are assigned to each electrode, the difference of which corresponds to the applied bias voltage, $\mathcal{V}$. We set the boundaries, $z=z_{L}, z_{R}$, deep in the left and right jellium electrodes, respectively, and the effective potentials are taken to be constant, $V_{L}$ and $V_{R}$, outside the boundary due to the absence of scattering there.

Scattering states of the system incident from the left or right are expressed as plane waves in multichannels, $\Psi^{L(R)}(\boldsymbol{r})$, which consist of a set of wave functions 
$\Psi_{m}^{L(R)}(\boldsymbol{r})$ incident from channels $m$ of the left (right) electrodes:

$$
\Psi^{L(R)}(\boldsymbol{r})=\left[\Psi_{1}^{L(R)}(\boldsymbol{r}), \Psi_{2}^{L(R)}(\boldsymbol{r}), \cdots, \Psi_{N}^{L(R)}(\boldsymbol{r})\right] .
$$

Hereafter we do not explicitly write the index L or R to simplify expressions except in the case of importance. The wave function $\Psi_{m}$ is expanded using plane waves in the $x$ and $y$ directions in the Laue representation,

$$
\Psi_{m}(\boldsymbol{r})=e^{i \boldsymbol{k}_{\|} \cdot \boldsymbol{r}_{\|}} \sum_{n} \psi_{n m}(z) e^{i \boldsymbol{G}_{\|}^{n} \cdot \boldsymbol{r}_{\|}},
$$

where $\boldsymbol{G}_{\|}^{n}$ is a set of two-dimensional reciprocal lattice vectors in the $\boldsymbol{r}_{\|}=(x, y)$ direction.

For the system without atoms, there is no interchannel scattering, i.e., $\psi_{n m}=0(n \neq m)$. The wave function $\Psi^{0}(\boldsymbol{r})$ is obtained by solving

$$
\left(E-\mathcal{H}_{0}\right) \Psi^{0}(\boldsymbol{r})=0 .
$$

The $z$ axis is discretized as $z_{p}$ with the width $\Delta z$, and the electronic states are easily solved self-consistently under the boundary conditions.

$$
\begin{aligned}
& \psi_{n n}^{0 L}(z)= \begin{cases}e^{i k_{z}^{n L} z}+r e^{-i k_{z}^{n L} z} & \left(z \leq z_{L}\right) \\
t e^{i k_{z}^{n R} z} & \left(z \geq z_{R}\right)\end{cases} \\
& \psi_{n n}^{0 R}(z)= \begin{cases}t^{\prime} e^{-i k_{z}^{n L} z} & \left(z \leq z_{L}\right) \\
e^{-i k_{z}^{n R} z}+r^{\prime} e^{i k_{z}^{n R} z} & \left(z \geq z_{R}\right)\end{cases}
\end{aligned}
$$

where

$$
k_{z}^{n L(R)}=\sqrt{\left(2 m / \hbar^{2}\right)\left(E-V_{L(R)}\right)-\left|\boldsymbol{k}_{\|}+\boldsymbol{G}_{\|}^{n}\right|^{2}} .
$$

The wave function of the total system, $\Psi(\boldsymbol{r})$, is obtained by solving the Lippmann-Schwinger equation

$$
\boldsymbol{\Psi}(\boldsymbol{r})=\mathbf{\Psi}^{0}(\boldsymbol{r})+\int d^{3} \boldsymbol{r}^{\prime} d^{3} \boldsymbol{r}^{\prime \prime} g\left(\boldsymbol{r}, \boldsymbol{r}^{\prime}\right) \Delta V\left(\boldsymbol{r}^{\prime}, \boldsymbol{r}^{\prime \prime}\right) \boldsymbol{\Psi}\left(\boldsymbol{r}^{\prime \prime}\right) .
$$

The electron density is obtained from the wave function. The Hartree potential is calculated by solving the Poisson equation under the boundary condition. The exchange-correlation potential is obtained by the local density approximation. We use the Ceperley-Alder form [8] parameterized by Perdew and Zunger [9]. Thus, a new effective potential is obtained. The calculation of the wave functions is repeated, and electronic states are calculated self-consistently. The current density is calculated as the probability current density of the wave function for the self-consistent solution. For a sufficiently large supercell compared with the size of the wire, we can use only the $\Gamma$ point, $k_{\|}=(0,0)$, to determine the transmission properties. In such a case the current per unit cell is simply expressed as the Landauer formula

$$
I=\frac{2 e}{h} \int_{E_{F}^{R}}^{E_{F}^{L}} \operatorname{tr}\left(\mathbf{T}^{\dagger} \mathbf{T}\right) d E,
$$

where $\mathbf{T}$ is the transmission matrix,

$$
\mathbf{T}_{n m}=\sqrt{\frac{k_{z}^{m R}}{k_{z}^{n L}}} \psi_{n m}\left(z_{R}\right) .
$$

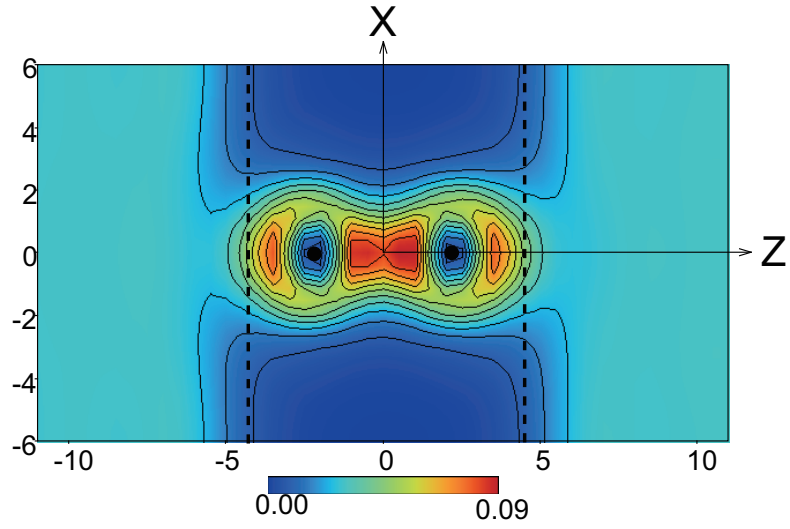

FIG. 1: Electron density distributions in the $x-z$ plane for a bias voltage of $3 \mathrm{~V}$. The red and blue regions indicate high and low density regions, respectively. The black circles indicate the position of Si atoms. The vertical dashed line indicates the edge of the semi-infinite jellium electrodes.

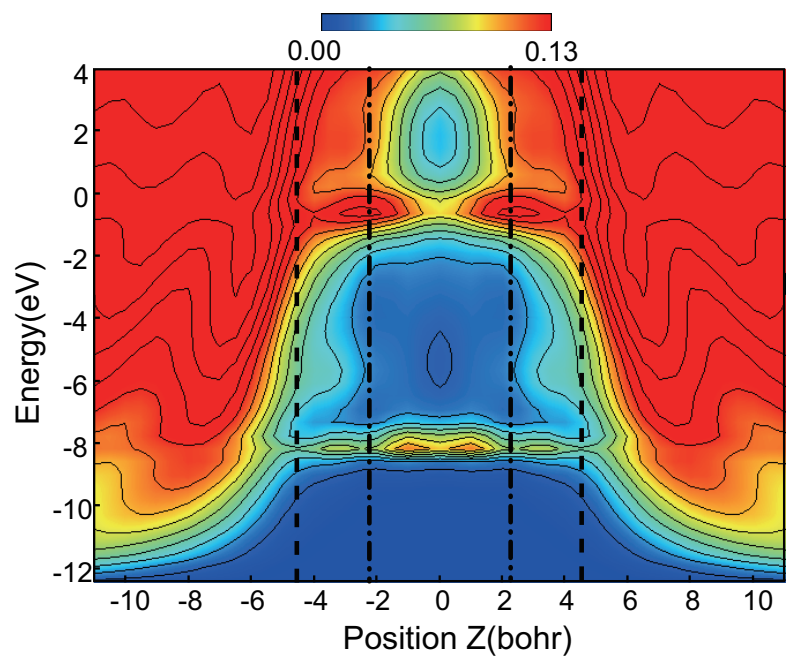

FIG. 2: Local density of states averaged in the $\mathrm{x}, \mathrm{y}$ plane. The red and blue regions indicate high and low density regions, respectively. The vertical dashed-dotted lines indicate the positions of Si atoms. The vertical dashed lines indicate the edges of the semi-infinite jellium electrodes.

The Hermitian matrix $\mathbf{T}^{\dagger} \mathbf{T}$ is diagonalized using unitary matrix $\mathbf{U}$ to transform the original channels into eigenchannels.

$$
\operatorname{tr}\left(\mathbf{T}^{\dagger} \mathbf{T}\right)=\operatorname{tr}\left(\mathbf{U}^{\dagger} \mathbf{T}^{\dagger} \mathbf{T} \mathbf{U}\right)=\sum_{n} T_{n}
$$

Therefore, the transmission properties are expressed using the sum of the transmission of the eigen channels, $T_{n}$

\section{RESULTS AND DISCUSSION}

Figure 1 shows electron density distributions in the $x-z$ plane for a bias voltage of $3 \mathrm{~V}$. The black circles indicate the positions of $\mathrm{Si}$ atoms. The red and blue regions indicate high and low electron density regions, respectively. The covalent bond is clearly seen between the $\mathrm{Si}$ atoms 


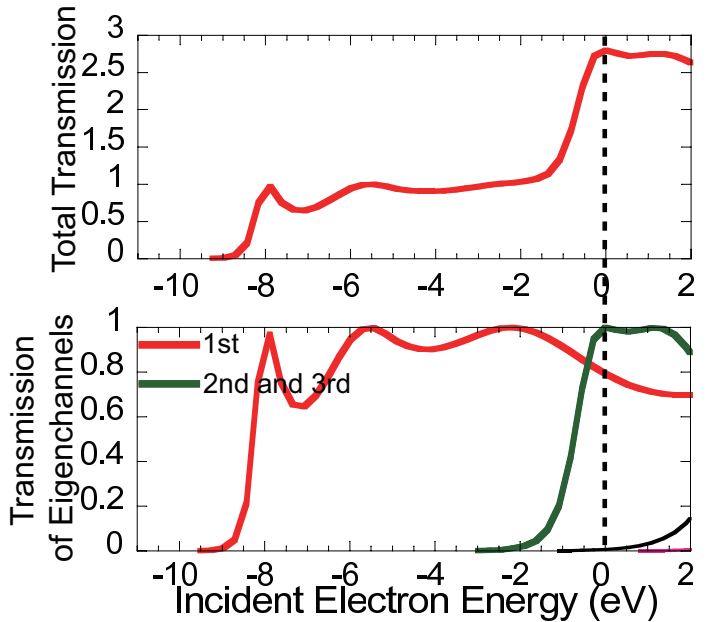

FIG. 3: The transmissions of eigen channels of the Si atom wire as a function of incident electron energy (lower) and the total transmission spectrum of eigen channels (upper). The applied bias is $0 \mathrm{~V}$. The origin of the energy is taken as $E_{F}^{L}=E_{F}^{R}=0$ (the vertical dashed line).

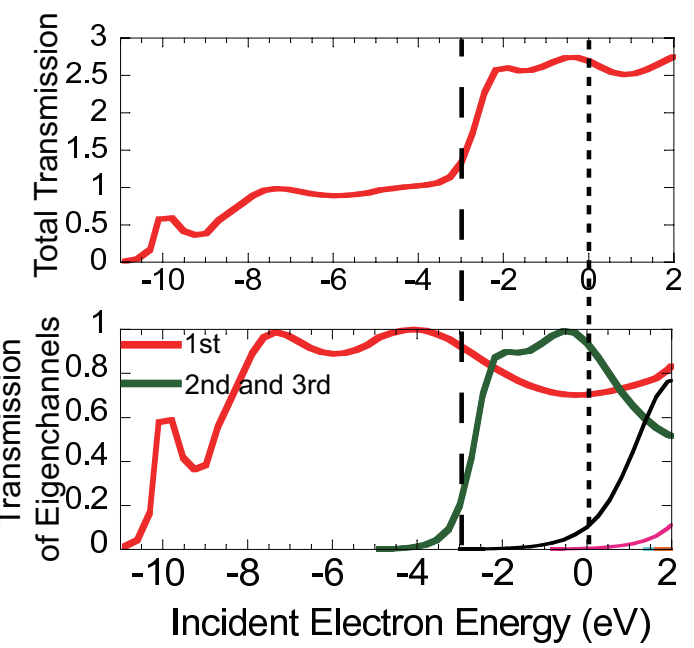

FIG. 4: The transmissions of eigen channels of the Si atom wire as a function of incident electron energy (lower) and the total transmission spectrum of eigen channels (upper). The applied bias is $3 \mathrm{~V}$. The origin of the energy is taken as $E_{F}^{L}=0$ (the vertical short dashed line). The vertical long dashed line indicates the $E_{F}^{R}$ for a applied bias of $3 \mathrm{~V}$.

with a slight polarization due to the bias voltages. The bonding nature between the atom and the metallic electrode is also seen, which could cause the electron scattering at the interface region between them.

Next, in order to investigate the effects of the metallic electrodes on the Si atom wire, we have analyzed the local density of state (LDOS) for a bias of $0 \mathrm{~V}$ (Fig. 2). The peaks at the energy of around $-8 \mathrm{eV}$ (green region) is seen in the Si atom wire region corresponding to the s orbital. LDOS has no zero value in all the energy region, although it takes minimum around $-5 \mathrm{eV}$. It is quite different feature from an infinite length of straight $\mathrm{Si}$ atom wire, the band structure of which has the band gap just below the Fermi energy [5]. This indicates additional states of the metal induced gap states due to the metallic electrode contacts.

Finally, we show the conduction channels as a function of the incident electron energy in lower panels of Fig. 3 and Fig. 4 for a bias of $0 \mathrm{~V}$ and $3 \mathrm{~V}$ respectively. the upper panels of Fig. 3 and Fig. 4 show the total transmission spectrum which is the sum of the transmission of each eigen channel for a bias of $0 \mathrm{~V}$ and $3 \mathrm{~V}$, respectively. The quantization of transmission spectrum is clearly seen because the $\mathrm{Si}$ atom wires behave as metallic due to the metallic electrode contacts [4]. For the bias of $3 \mathrm{~V}$, The energy region between the vertical dashed lines corresponds to the bias window. The three channels contribute to the transport in the $\mathrm{Si}$ atom wire; the first channel has the characters of $s-p_{z}$ states and the second and third channels have the characters of the degenerate $p_{x}$ and $p_{y}$ states, respectively. These three channels open in the bias window under the finite bias voltage, which is different from $\mathrm{Al}$ wires $[6]$.

\section{SUMMARY}

We presented a first-principles calculations of electron transport in a $\mathrm{Si}$ atom wire sandwiched between semiinfinite jellium electrodes under the finite bias voltage. Electronic states and transport properties were calculated using the Lippmann-Schwinger equation using the Laue representation in the framework of the density functional theory. The Si atom wire behaves as metallic due to the metal induced gap state caused by metallic electrode contacts. Using eigen channel decompositions, we found that the three channels contributing to the transport are open in the bias window.

\section{Acknowledgments}

This work was supported in part by a Grant-in-Aid for Scientific Research and the Next Generation Super Computing Project, Nanoscience Program from the Ministry of Education, Culture, Sports, Science and Technology of Japan. Numerical calculations were performed using the SR11000 at ISSP, University of Tokyo and also carried out under the "Interdisciplinary Computational Science Program" in Center for Computational Sciences, University of Tsukuba.
[1] For a review, see, e.g., N. Agraït, A. L. Yeyati, and J. M. van Ruitenbeek, Phys. Rep. 377, 81 (2003).

[2] T. Kizuka, Y. Takatani, K. Asaka, and R. Yoshizaki, Phys. Rev. B 72035333 (2005).

[3] T. Iwanari, T. Sakata, Y. Miyake, S. Kurokawa, and A
Sakai, J. Appl. Phys. 102114312 (2007).

[4] J. L. Mozos, C. C. Wan, G. Taraschi, J. Wang, and H. Guo, Phys. Rev. B 564351 (1997).

[5] S. Okano, K. Shiraishi, and A. Oshiyama, Phys. Rev. B 69045401 (2004). 
[6] N. Kobayashi, M. Aono, and M. Tsukada, Phys. Rev. B 64, 121402 (2001).

[7] For a method using the three-dimensional plane wave basis set, cf. N. D. Lang, Phys. Rev. B 52, 5335 (1995); M. Di. Ventra and N. D. Lang, Phys. Rev. B 65045402 (2002).

[8] D. M. Ceperley and B. J. Alder, Phys. Rev. Lett. 45, 566 (1980).
[9] J. P. Perdew and A. Zunger, Phys. Rev. B 23, 5048 (1981).

[10] N. D. Lang and Ph. Avouris, Phys. Rev. Lett. 84, 358 (2000).

[11] K. Hirose, N. Kobayashi, and M. Tsukada, Phys. Rev. B 62, $245412(2004)$. 